\title{
Approximate Analytical Technique to Design Reflectarray Antenna
}

\author{
Hesham M. Yamani' ${ }^{1}$, Ahmed M. Attiya ${ }^{1}$, Alaa K. Abdelmageed², \\ ${ }^{1}$ Microwave Engineering Department, Electronics Research Institute, Giza, Egypt \\ ${ }^{2}$ Department of Engineering Mathematics and Physics, Cairo University, Giza, Egypt \\ *eng.hesham. yamani@gmail.com
}

\begin{abstract}
This paper presents an analysis and design for a reflectarray antenna composed of an array of rectangular patches printed on a grounded dielectric slab. A simple analytical technique based on equivalent surface impedance is used to determine the reflection of the elements in reflectarray antenna. This equivalent surface impedance is obtained analytically in a closed form. The effect of the angle of incidence on each element in the reflectarray is included in calculations. To author's knowledge, this property has not been included in previous analysis techniques of reflectarray antenna.
\end{abstract}

\section{Introduction}

High gain antennas are important for different applications like satellite communication systems, point to point microwave links and radar applications. High gain can be obtained by using reflector antennas, lens antennas or antenna arrays. The main disadvantages of the first two types are the bulky structures and the heavy weight. The main disadvantage of the third type is the large losses in the feeding network of large arrays. Berry et al. [1] suggested a combination between antenna arrays and reflector antenna. They introduced a planer reflectarray with phase distribution similar to the corresponding one on the aperture of reflector antenna due to the same feed. This phase distribution can be obtained in the case of a planar reflectarray by adjusting the dimensions of the patches of the reflectarray.

Full wave analysis of the microstrip reflectarray was introduced by Pozar [2]. In this case each element of the reflectarray is represented as an element in an infinite array. A single element with periodic boundary conditions is used to model the reflection of this element for different dimensions. Based on this analysis and the required phase distribution the size of each element in the reflectarray is determined. The analysis of a single element inside periodic boundary conditions can be obtained numerically by using the method of moments to solve electric field integral equation, the finite difference time domain method or the finite element method [3-4]. The common disadvantage of these techniques is the required computational time. Thus, these methods are usually used to determine the reflection of the element at normal incidence only and use the result for all the elements in the reflectarray. For actual case, the incident field from the source to each element on the reflectarray antenna has its own angle of incidence which is different from other elements. Direct numerical calculation of each element with its angle of incidence would require huge computational time and storage. Capozzoli et al. [5-6] introduced an algorithm for reflectarray synthesis based on MoM. Their algorithm takes into consideration the actual incidence angle. They applied numerical acceleration by using parallel programming based on Graphics Processing Units to overcome the required large computational time.

Recent approaches in reflectarrays are based on closely spaced elements [8-9] or sub-wavelength elements [10] to increase the operating bandwidth of the reflectarray. For infinite arrays of these closely spaced and sub-wavelength elements of simple canonical shapes, the reflection coefficients can be formulated analytically in simple closed forms by using analytical techniques [11]. The advantage of these analytical techniques is that they can include the angle of incidence without additional computational cost. The analytical form of the reflection coefficient of the element is formulated as an equivalent surface impedance. To include the effect of the substrate, an equivalent transmission line network is used. The complete network based on this surface impedance and the transmission line network is used to obtain the reflection coefficient for any angle of incidence. Albertsen [7] proposed a similar analytical method based on calculating magnetic currents at the gaps between rectangular patches. The radiated fields are obtained by using Love's equivalence principle.

In this paper, a new analytical representation is used to determine the sizes of the different patches on the reflectarray surface which correspond to the required phase distribution on the aperture of the reflectarray. The dimensions of each element are calculated by using simple analytical form which is function of the location of the element, the angle of incidence and the required phase at this location. The design is verified by simulating the complete reflectarray using CST [12].

It should be noted that the total radiation pattern of a reflectarray is a function of both amplitude and phase distribution of the scattered fields from the aperture of the reflectarray. The phase distribution at each point is mainly a function of the size and the shape of the reflecting element at this point which is the main target of the present paper. However, the amplitude distribution of the scattered wave at the aperture is mainly a function of the feeding structure and its location. Although, the present results are limited to simple cases of a pencil beam, this analysis can also be applied for other conditions by using the corresponding appropriate amplitude and phase distributions to control the shape of the beam and the sidelobe level. However, this is 
not the main target of the present paper.

The organization of the paper is as follows; Sec. 2 presents the basic theory of the analytical technique for designing the element of the reflectarray. Sec. 3 represent the results and discussions of the calculated far field of the reflectarray. Also a comparison between the reflectarray antenna and parabolic reflector is represented. Finally conclusion is presented in $\mathrm{Sec} 4$.

\section{Theory}

\subsection{Basic Theory of reflectarray Antenna}

The proposed reflectarray is assumed to be composed of square patches on a grounded dielectric slab as shown in Fig. 1. The feeding structure is a simple horn antenna located at the focal point of the reflectarray. The required phase distribution on the surface of the reflectarray is determined by the condition [2]:

$$
k_{0}\left(R_{i}-\mathbf{r}_{i} \cdot \mathbf{r}_{0}\right)-\psi_{i}=2 \pi N
$$

where $\psi_{i}$ is the required phase of the reflected field at the point $\mathbf{r}_{i}$ on the aperture of the reflectarray to produce a main beam at the direction of the unit vector $\mathbf{r}_{0}$ and $R_{i}$ is the distance from the feeding point to the required point on the aperture of the reflectarray. This phase distribution at each point on the surface of the reflectarray is obtained by adjusting the size of the square patch at this point. The dimension of the unit cell is assumed to be constant. The analysis of the reflection coefficient of each cell is the key point in the design procedure.

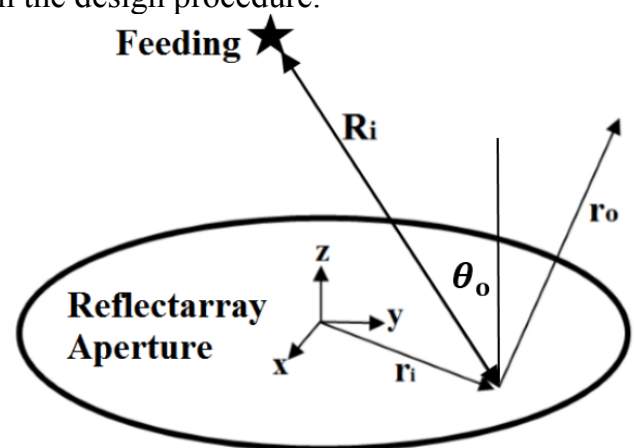

Figure 1: Reflection from a general point on a reflectarray aperture.

The present analysis is based on approximating each cell to be nearly embedded inside an infinite array of such cells. This approximation can be accepted since the variation between adjacent cells is usually very small compared to wavelength in a close area. Another approach based on macro cell configuration can also be used to present the effect of a small group of scattering elements on the reflectarray structure [6]. However, this approach may not be directly suitable in the present case to obtain the equivalent surface impedance of each element. Thus, the present analysis would be limited on the infinite array approach.

\subsection{Equivalent Impedance Method of Infinite Periodic Patches}

Periodic conducting patches, as shown in Fig. 2, can be represented as an equivalent surface impedance by using averaged boundary conditions (ABC). For the case of periodic patches on a grounded dielectric slab, the problem can be modeled as a short-circuited transmission line section loaded by the surface impedance of the patches as shown in Fig. 3. The corresponding characteristic impedances of these transmission line sections depend on the polarization of the incident wave and the angle of incidence as follows [13]:

$$
\begin{aligned}
& Z_{n}^{T E}=\eta_{n} / \cos \theta_{n} \\
& Z_{n}^{T M}=\eta_{n} \cos \theta_{n}
\end{aligned}
$$

where $\eta_{n}$ is the characteristic impedance of the medium $n$. $n=0$ corresponds to free space and $n=1$ corresponds to the dielectric slab. Here $\theta_{0}$ is the angle of incident wave and $\theta_{1}$ is the angle of transmitted wave inside the dielectric slab. The equivalent surface impedance of the periodic patches is a function of the periodic cell, the dimensions of the patch, the angle of incidence and the polarization of the incident wave as follows [13]:

$$
\begin{aligned}
& Z_{g}^{T E}=-j \eta_{0} /\left\{2 \alpha\left(1-\left(\sin ^{2} \theta_{0}\right) / 2\right)\right\} \\
& Z_{g}^{T M}=-j \eta_{0} /\{2 \alpha\}
\end{aligned}
$$

where $\alpha$ is the grid parameter which is given by:

$$
\alpha=k_{\text {eff }} D / \pi \cdot \ln (1 / \sin (\pi w / 2 D))
$$

$D$ is the unit cell size and $\mathrm{w}$ is the spacing between the patches as shown in Fig. 2. $k_{\text {eff }}=k_{0} \sqrt{\varepsilon_{\text {eff }}}, \varepsilon_{\text {eff }}=$ $\left(\varepsilon_{r}+1\right) / 2$ is the relative effective permittivity and $\mathrm{k}_{0}$ is the wave number in free space. Equations (4) and (5) are valid only if $k_{e f f} D<2 \pi$ [13]. It should be noted from (5) that equivalent surface impedance of periodic patches is independent on the angle of incidence for the case of TM polarization.

Based on Eq. (4) and (5) and the equivalent transmission line circuit one can obtain the equivalent surface impedance of a grounded dielectric slab loaded by periodic patches for both TE and TM polarization as follows:

$$
\begin{aligned}
& Z_{\text {total }}^{T E}=Z_{g}^{T E} / / j Z_{1}^{T E} \tan \kappa_{1} t \\
& Z_{\text {total }}^{T M}=Z_{g}^{T M} / / j Z_{1}^{T M} \tan \kappa_{1} t
\end{aligned}
$$

where $\mathrm{t}$ represents the substrate thickness and $\kappa_{1}=$ $k_{1} \cos \theta_{1}$ is the normal component of the propagation constant inside the dielectric slab.

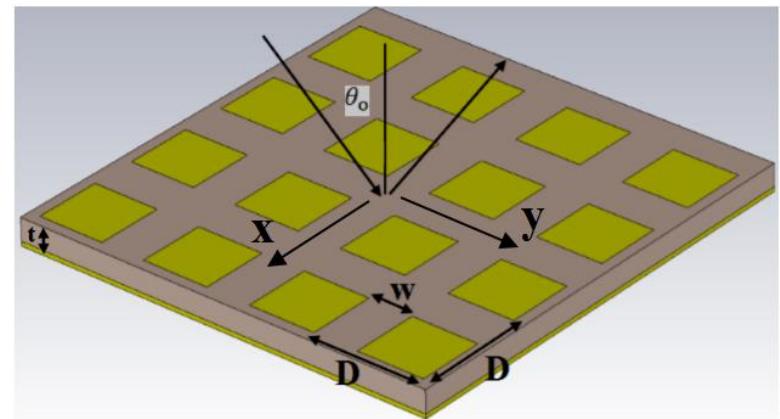

Figure 2: Geometry of scattering by periodic patched on a grounded dielectric slab.

The reflection coefficient due to the periodic patches on the grounded dielectric slab can be obtained in closed forms as follows [13]: 


$$
\begin{gathered}
\Gamma^{T E}=\frac{Z_{\text {total }}^{T E}-Z_{o}^{T E}}{Z_{\text {total }}^{T E}+Z_{o}^{T E}} \\
\Gamma^{T M}=\frac{Z_{\text {total }}^{T M}-Z_{o}^{T M}}{Z_{\text {total }}^{T M}+Z_{o}^{T M}}
\end{gathered}
$$

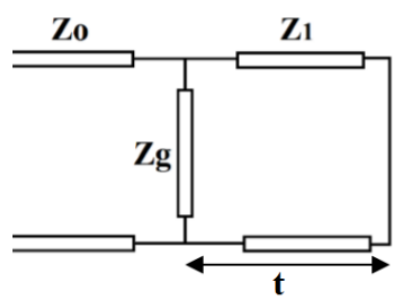

Figure 3: Equivalent transmission line network for a grounded dielectric slab loaded by a surface impedance.

To test the validity of the above two equations, a comparison between analytical and numerical solutions of reflection coefficient of a grounded dielectric slab loaded by periodic square patches is presented. The numerical solution is based on MoM analysis as discussed in [2].The slab is assumed to be Rogers 6010 with dielectric constant $\varepsilon_{r}=10.2$ and dielectric thickness $t=1 \mathrm{~mm}$. The authors examined other values of dielectric constants. It is found that lower values of dielectric constant like 2.2 cannot obtain the required phase range for the proposed scattering elements. The periodic cells are assumed to be square of a periodic length $D=5 \mathrm{~mm}$. The nominal size of the square patch is assumed to be $L_{0}=3.6 \mathrm{~mm}$. The size of the patch is varied to be $L=L_{0}+\Delta L$ where $\Delta L / L_{0}$ varies from -0.35 to 0.35 as shown in Fig. 4. The operating frequency is assumed to be $10 \mathrm{GHz}$ and the incident angle in this case is 20 degrees. Figure 4 shows a comparison between the analytical solution and the numerical solution. It can be noted the excellent agreement between the two solutions for both TE and TM polarizations.

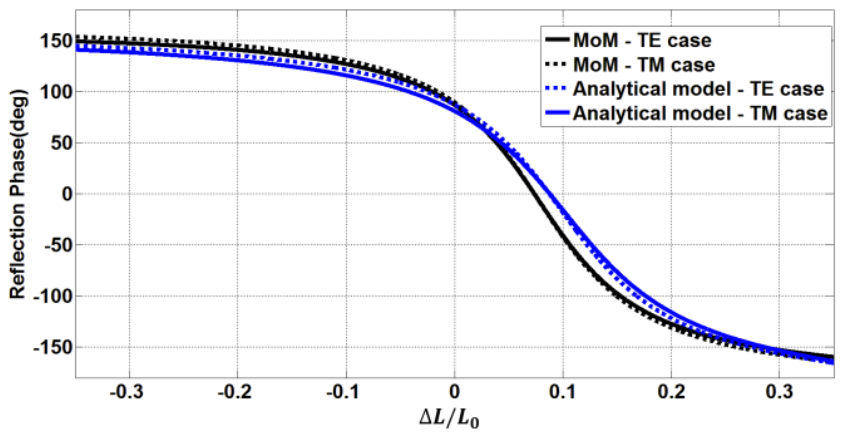

Figure 4: Phase of the reflected field versus patch size

Based on the above result, it can be concluded that Eq. (9) and (10) can be used directly to obtain the complex reflection coefficients for both TE and TM incident plane wave on a periodic array of square patches printed on a grounded dielectric slab. These equations include the angle of incidence. Thus, by inserting these two equations in the design procedure of the reflectarray it would be possible to obtain the required dimension of the patch at each cell according to the polarization and angle of incidence at the location of this patch. This property is not included in previous design procedures which are based on MoM analysis or other similar numerical techniques.

\section{Design of a Reflectarray based on Analytical Reflection Coefficient}

In this section, the above mentioned theory is used to design an X-band reflectarray antenna based on square patches on a grounded dielectric slab. The operating frequency is assumed to be $10 \mathrm{GHz}$. The parameters of the grounded dielectric slab and the periodic patches are the same as mentioned in the above analysis of Fig. 4. The reflectarray consists of $60 \times 60$ square patches which corresponds to a square aperture of length $10 \lambda$ at the proposed operating frequency. The focal to edge length ratio of this reflectarray is assumed to $f / D=1$. A conical horn antenna at the focal point is used as the feeding antenna for this reflectarray. The radiation pattern of this horn antenna is nearly equivalent to $\cos ^{4} \theta$ [14]. For such $f / D$ ratio and the proposed feeding antenna, the subtended angle of the reflectarray by the feeding beam is 26.5 degrees and the amplitude taper at the reflectarray edges is nearly $-5 \mathrm{~dB}$.

Based on the length of the square aperture and the $f / D$ ratio, the required phase distribution on the aperture of the reflectarray is obtained by using Eq. (1) as shown in Fig. 5. The next problem is to determine the sizes of the patches at each cell on the aperture of the reflectarray. In the present technique, the angle of incidence and the polarization of the incident quasi-plane wave on each cell individually is taken into consideration. Then these parameters are used into (9) and (10) to find out the length of the patch which introduces the required phase at this angle of incidence and polarization. Based on this procedure, the sizes of the patches on all cells are obtained.

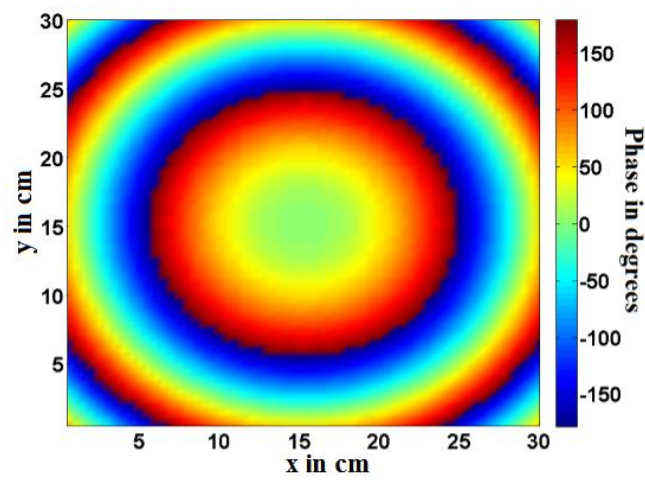

Figure 5: The required phase distribution on the aperture of the reflectarray. The operating frequency is $10 \mathrm{GHz}$, $d=30 \mathrm{~cm}$ and $f / D=1$.

The obtained geometry of the complete reflectarray is simulated by using full wave electromagnetic simulator (CST) to obtain its gain as a function of frequency. Figure 6 shows the obtained gain in this case. It is found that the peak gain is shifted towards a lower frequency at nearly $9 \mathrm{GHz}$. In [13] it is mentioned that this shift may be explained due to the increase of effective capacitance of the closely spaced elements. Hence, the resonance frequency will be lower than the expected value. To compensate this frequency shift, a 
correction factor $m$ for $\mathrm{w}$ is added to the grid parameter in (6) as follows:

$$
\alpha_{m}=k_{e f f} D / \pi \cdot \ln (1 / \sin (\pi m w / 2 D))
$$

Different values for $m$ are studied to obtain the optimum value which introduce the peak gain at the proposed design frequency. According to the different results, the optimum value of $m$ is found to be nearly 0.7 . Figure 7 shows the gain of the reflectarray antenna after using the modified grid parameter. It can be noted that the peak gain in this case is about $28 \mathrm{~dB}$ at the frequency range from 10 to $10.5 \mathrm{GHz}$ which is good compared to expected ideal maximum gain for this aperture area which should be about $31 \mathrm{~dB}$ according to the relation

$$
G_{\max }=4 \pi A / \lambda^{2}
$$

where $\mathrm{A}$ is the physical area of the radiation aperture. The losses in this case can be attributed due to spillover and amplitude taper efficiencies [2].

The same reflectarray is designed by using phase distribution based on normal incidence only as in the previously published analyses. The obtained gain of this reflectarray is compared with the corresponding gain of the present analysis as shown in Fig. 8. It can be noted that the reflectarray of the present analysis has $1 \mathrm{~dB}$ gain bandwidth of $12.43 \%$ while the reflectarray based on normal incidence analysis only has $1 \mathrm{~dB}$ gain bandwidth of $9.79 \%$.

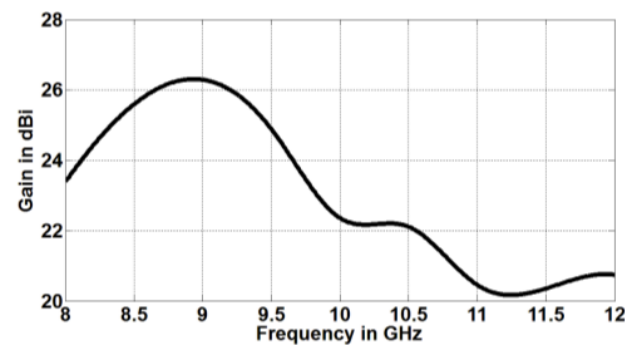

Figure 6: Calculated antenna gain for initial design using the analytical technique.

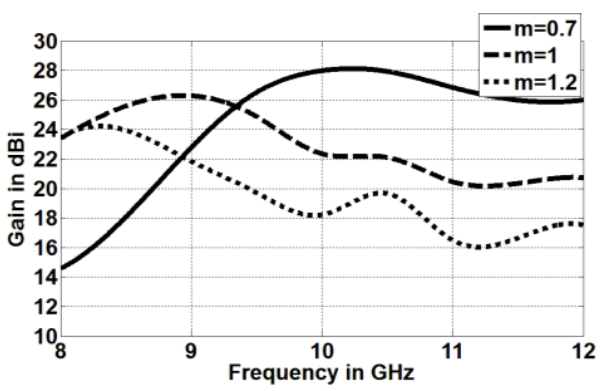

Figure 7: Calculated antenna gain for different values of correction coefficient $m$.

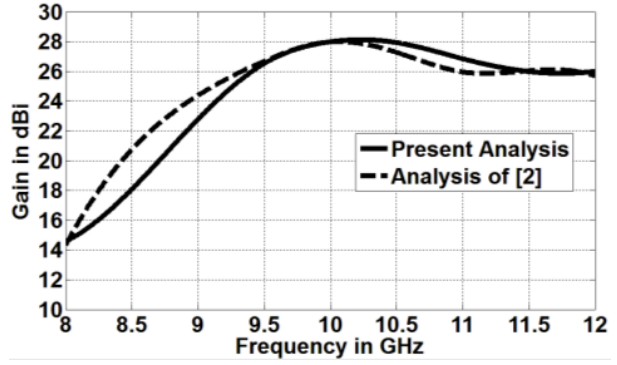

Figure 8: Comparison between calculated antenna gains of two reflectarray designs. The first design is based on analysis of the elements by using normal incidence only and the second design is based on the analysis of each element with its actual angle of incidence.

To compare this reflectarray antenna with the corresponding parabolic reflector antenna, we reduced the dimensions of the designed reflectarray to be of a circular aperture as in the case of the reflector antenna. The antenna gain of a designed circular reflectarray is compared with the corresponding gain of a parabolic reflector antenna with the same parameters; diameter, $f / D$ ratio and feed. Figure 9 shows this comparison. It can be noted that the present circular reflectarray has a gain of $26.74 \mathrm{dBi}$ at $10 \mathrm{GHz}$ while the parabolic reflector has a gain of $27.42 \mathrm{dBi}$ at the same frequency. It can also be noted that the gain of the reflector antenna is increasing by increasing the frequency. However, the gain of the reflectarray antenna is increasing up to about $10 \mathrm{GHz}$ then it decreases at higher frequencies. This can be explained due to the narrow band of the appropriate phase shift distribution of the reflectarray's elements.

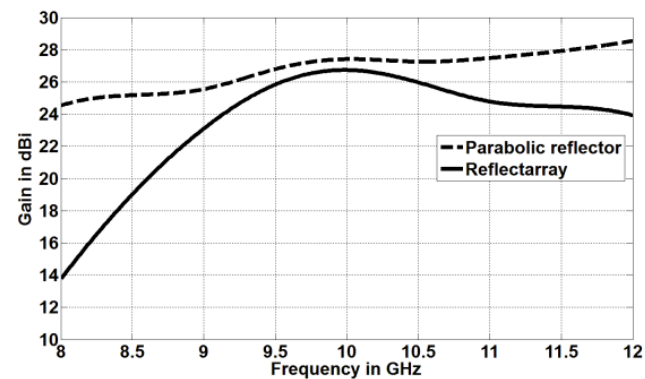

Figure 9: Comparison between the gain of a parabolic reflector antenna and the designed reflectarray.

\section{Conclusions}

A simple and fast analytical technique is used to model the elements of reflectarray antenna. This model includes the angle of incidence and the polarization of the incident ray on each element of the reflectarray antenna. This property improves the accuracy of the design of the total reflectarray antenna. It is shown that the designed reflectarray by using this technique has a better gain bandwidth compared with the corresponding reflectarray designed by using normal incidence analysis. The result of the designed reflectarray is also found to be with comparable properties in its operating bandwidth compared with the corresponding reflector antenna. The present analysis is limited to square patches on 
a grounded dielectric slab. However, the basic theory can be modified for other configurations. This point is under investigation.

\section{Acknowledgements}

The Authors would like to thank Prof. Essam Eldiwany for his valuable comments and suggestions.

\section{References}

[1] D. Berry, R. Malech, and W. Kennedy, "The reflectarray antenna," IEEE Trans. Antennas propag., 11: 645-651, 1963.

[2] D. M. Pozar, S. D. Targonski, and H. D. Syrigos, "Design of millimeter wave microstrip reflectarrays," IEEE Trans. Antennas Propag., 45: 287-296, 1997.

[3] D. Cadoret, A. Laisne , M. Milon , R. Gillard , and H. Legay, " FDTD analysis of reflectarray radiating cells, " IEEE/ACES International Conference on Wireless Communications and Applied Computational Electromagnetics, pp. 853 - 856 , 2005.

[4] I. Bardi , R. Remski , D. Perry, and Z. Cendes, " Plane wave scattering from frequency selective surfaces by finite element method ," IEEE Trans. Magn., 38: 641 - 644, 2002.

[5] A. Capozzoli, C. Curcio, A. Liseno, G. Toso, "Phase-only synthesis of flat aperiodic reflectarrays", Progress In Electromagnetics Research., 133: 53 - 89, 2013.

[6] A. Capozzoli, C. Curcio, A. Liseno, M. Migliorelli, G. Toso, "Analysis of a Periodic Reflectarray by Using FEKO", Proc. of the 29th Int. Rev. of Progr. in Appl. Comput. Electromagn., Monterey, CA, pp. 387-392 2013.

[7] N.C. Albertsen, "Analysis of reflectarrays using field synthesis," Proc. of the 33rd ESA Antenna Workshop on Challenges for Space Antenna Syst., ESTEC, Noordwijk, The Netherlands, 2011.

[8] D. M. Pozar, "Wideband reflectarrays using artificial impedance surfaces," Electron. Lett., 43: 148-149, 2007.

[9] P. Nayeri, F. Yang, and A. Z. Elsherbeni ,"Bandwidth improvement of reflectarray antennas using closely spaced elements" Progress In Electromagnetics Research C, 18: 1929, 2011.

[10] J. Ethier, M. R. Chaharmir and J. Shaker," New developments in reflectarray research at the communications research center canada (CRC)" Antenna Technology and Applied Electromagnetics, 15th International Symposium, 2012.

[11] S. A. Tretyakov, Analytical Modeling in Applied Electromagnetics, Artech House, 2003.

[12] CST microwave studio, www.cst.com

[13] G. Goussetis, D. Lioubtchenko, A.V. Räisänen, and S. A. Tretyakov, "Simple and accurate analytical model of planar grids and high-impedance surfaces comprising metal strips or patches" IEEE Trans. Antennas propag., 56:1624-1632, 2008

[14] C. A. Balanis, Antenna Theory: Analysis and Design, 2nd Edition, Wiley, Chichester, 1997. 